\title{
Remuneration and Tariffs in the Context of Virtual Power Players
}

\author{
Catarina Ribeiro ${ }^{1,2}$, Tiago Pinto ${ }^{1}$, Zita Vale ${ }^{1}$, José Baptista ${ }^{2}$ \\ ${ }^{1}$ GECAD - Research Group on Intelligent Engineering and Computing for Advanced Innova- \\ tion and Development, Institute of Engineering, Polytechnic of Porto (ISEP/IPP), Portugal/ \\ ${ }^{2}$ UTAD - Universidade de Trás-os-Montes e Alto-Douro \\ acrib@isep.ipp.pt, tmcfp@isep.ipp.pt,zav@isep.ipp.pt, baptista@utad.pt
}

\begin{abstract}
Power systems have been through deep changes, with their operation in the scope of competitive electricity markets (EM) and the increasingly intensive use of renewable energy sources and distributed generation. This requires new business models able to cope with the new opportunities. Virtual Power Players (VPPs) are a new player type which allows aggregating a diversity of players (distribution Generation, storage units, electrical vehicles, and consumers) to participate in the markets and to provide a set of new services promoting generation and consumption efficiency and to improving players' benefits. A major task of VPPs is the remuneration of generation and of the services (e.g. market operation costs, and energy reserves) as well as charging energy consumption. This $\mathrm{PhD}$ research will contribute by developing fair and strategic remuneration and tariff methodologies, able to allow efficient VPP operation and VPP goals accomplishment in EM.
\end{abstract}

\section{1.. Problem statement and related work}

The electricity sector has been completely revolutionized by the emergence of liberalized EM, characterized by many changes in operation rules, increase in competition and profound changes in the participant entities. The restructuring was performed so that the competitiveness could be increased, but it had exponential implications in markets complexity and unpredictability [1]. Much like EM subsystems of the main network are rapidly evolving into a reality, coordinating these entities is a huge challenge that requires the implementation of distributed intelligence, potentiating the concept of Smart Grid (SG) [1, 2]. However, inefficient resource management should be overcome by adequate optimization methods [3]. Player aggregating strategies allows players gaining technical and commercial advantages, individuals can achieve higher profits due to specific advantages of a mix of technologies to overcome disadvantages of some technologies. The aggregation of players gives rise to the concept of Virtual Power Player (VPP) [4]. VPPs aggregate different types of resources with individual goals; VPPs should conciliate all players in a common strategy able to enable each player to pursuit its own objectives [5]. Potential benefits will depend on the efficient operation in the market and the remuneration of aggregated players. Important developments concerning EM players modelling and simulation including decision-support capabilities can be widely found in the literature [4-6]. This $\mathrm{PhD}$ main goal is to develop remuneration and tariff methodologies in the scope of a VPP. The lack of tariff and remuneration definition methods to enable the compensation of prosumers in a EM environment, fairly and appropriately, is one of the gaps that this study will overcome.

This work is supported by FEDER Funds through COMPETE program and by National Funds through FCT under the project UID/EEA/00760/2013. 


\section{Proposed approach, preliminary results and reflections}

This work addresses the identified gaps in the literature by proposing the RemT (Remuneration and Tariffs) methodology. The results of the proposed RemT process are used in a learning algorithm to improve the quality of the remuneration process, with consequential implications to the market bidding process. The establishment of remuneration and tariffs is based on the identification of players' types and on the development of contract models for each player type. The players modelling considers the operation and market context. The terms for new contracts and best strategies for each context are determined by means of machine learning based methods. The first part of the work is related with consumer's classification of player's profiles. Data mining methodology was used, based on the application of a clustering process, which groups the typical load profile of the consumers of a SG according to their similarity. The separation of consumers in different groups allows proposing specific consumption tariffs to each group, so that consumers' load profile is taken into account to meet the objectives of the SG aggregator. This methodology is tested using a real smart grid with 82 consumers, that includes several consumers of different types (residential and commerce). This work proposes a clustering methodology that uses different data normalization methods and a new customized normalization method has been introduced [7]. Preliminary results demonstrated the advantages of data mining methodologies, based on the application of clustering process to group typical load profiles according to their similarity to allow proposing specific consumption tariffs to each group, so that consumers load profile is taken into account to meet the objectives of the SG aggregator. This work allows the development of a tool that provides a decision support for VPP definition of best tariff and remuneration to apply to each aggregated player, RemT.

\section{References}

1. M. Shahidehpour et al.,"Market Operations in Electric Power Systems: Forecasting, Scheduling, and Risk Management", Wiley-IEEE Press, pp. 233-274, 2002

2. Blumsack S and Fernandez A."Ready or not, here comes the smart grid!"Energy. 2012; 37(1):61-8

3. Sousa T. et al.,"Intelligent Energy Resource Management Considering Vehicle-to-Grid: A Simulated Annealing Approach," Smart Grid, IEEE Trans. on, 3, 535-542, 2012

4. Z. Vale et al.,"MASCEM - Electricity markets simulation with strategically acting players", IEEE Intelligent Systems, vol. 26, n. 2, Special Issue on AI in Power Systems and Energy Markets, 2011

5. T. Pinto, et al, "Multi-Agent Based Electricity Market Simulator With VPP: Conceptual and Implementation Issues", 2009 IEEE PES General Meeting, 2009

6. V. Koritarov "Real-World Market Representation with Agents: Modeling the Electricity Market as a Complex Adaptive System with an Agent-Based Approach", IEEE Power \& Energy magazine, pp. 39-46, 2004

7. C. Ribeiro et al. "Customized Normalization Method to enhance the Clustering process of Consumption Profiles" Advances in Intelligent Systems and Computing, 7th International Conference on Ambient Intelligence (ISAMI'16), 2016. 